\title{
A CASE OF COMPLETE HEART BLOCK DUE TO INTENTIONAL INGESTION OF PLANT EXTRACTS
}

\author{
K. Nagendra Prasad, K. Lavanya.
}

\begin{abstract}
:
Cardiac glycosides are widely available in botanic products and other naturally occurring substances worldwide. Accidental consumption of it leads to digitalis toxicity with varied systemic manifestations. We describe a case of consumption of extract of leaves of the Indian rubber vine plant (Crytostegia grandi flora) which led to gastrointestinal, cardiac, electrolyte, and hematological disturbances.
\end{abstract}

\section{INTRODUCTION:}

Cardiac glycosides are widely available in some botanic products and other naturally occurring substances. Cryptostegia grandiflora, also known as the Indian rubber vine is said to contain cardiac glycosides responsible for producing a digitalis-like toxicity on consumption of its leaves. Digitalis toxicity produces a toxidrome characterized by gastrointestinal, neurologic, electrolyte, hematologic and cardiac manifestations [1].

\section{CASE REPORT:}

A $39 \mathrm{yr}$ old female patient was brought to the hospital with complaints of decreased urine output and altered sensorium. She was treated in outside hospital where she admitted with recurrent green colored vomitings and pain abdomen following Ingestion of an unknown plant leafs intentionally. Following stomach wash, Intravenous fluids, antiemetics she improved symptomatically but patient developed tachycardia which is documented in ECG taken outside. It is regular narrow complex tachycardia probably Atrial tachycardia(fig 2).

Article received on 22 MAR 2017, published on 30 APR 2017.

K.Nagendra Prasad ${ }^{1}$, K. Lavanya ${ }^{2}$

${ }^{1}$ Senior Resident, Department of Cardiology,NIMS,India

2DNB Resident, , Department of GM, ST Martha's.

Corresponding Author: K.Nagendra Prasad

Email: nagendra.kulari@gmail.com
Fig. 2: ECG showing SVT

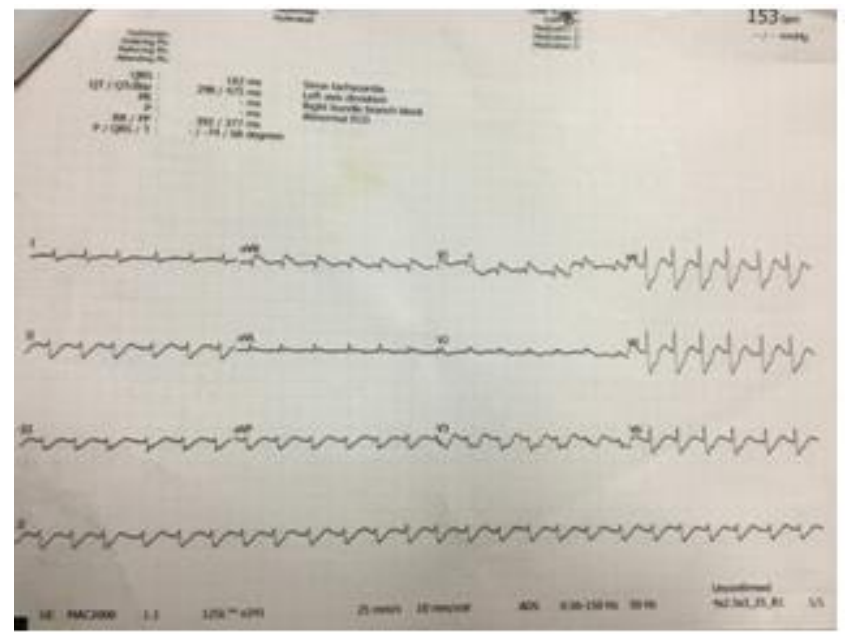

Later on the same day, she developed cardiac arrest, after resuscitation, it converted to complete heart block (Fig1)

Fig. 1: ECG showing complete heart block

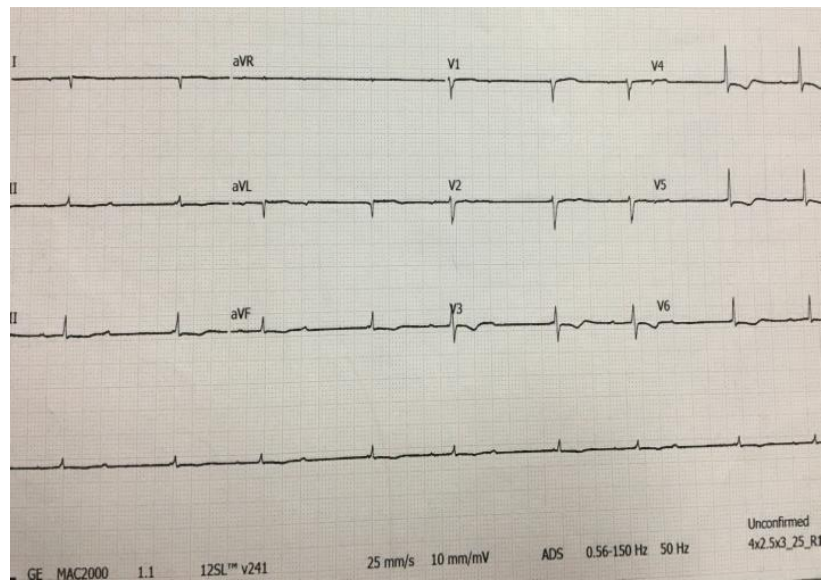

with escape heart rate of $50 / \mathrm{min}$ for which she was kept on the temporary pacemaker. Paced rhythm showed in (fig 3). 
Fig 3: ECG showing paced rhythm

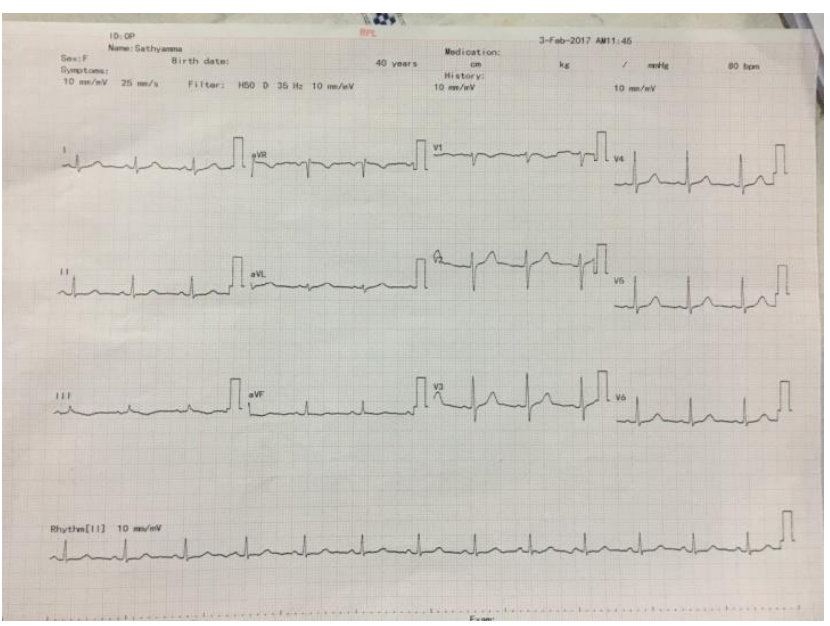

Patient had hypotension and respiratory compromise which was treated with mechanical ventilation and inotropes. In view of all these problems, she was referred to our hospital. At the time of arrival, she was conscious and there is no respiratory distress. Blood pressure is maintaining well with low dose inotrope. On the next day, she was extubated and inotropes were weaned off. Complete heart block persisted for one week. The patient developed acute kidney injury, (serum creatinine - $2.4 \mathrm{mg} / \mathrm{dl}$ ) which was managed conservatively following Nephrologist advice. There is no hyperkalemia. During ICU stay we noticed thrombocytopenia (PLT - 60000) without major or minor bleeding manifestations. It recovered to normal within one week. Complete heart block recovered to normal sinus rhythm After 10 days of poisonous tree extract ingestion. In view of a possible digitalis intoxication, serum digoxin levels were measured which were $6 \mathrm{ng} / \mathrm{ml}$ (normal range: 0.8 to $2 \mathrm{ng} / \mathrm{ml}$ ).

On detailed examination of the leaves the ones that the patient had consumed, which the patient attenders brought, it was identified to be the leaves of Cryptostegia Grandiflora also called as the Indian rubber wine which is the source of cardiac glycosides (Fig 4).
Fig4: Ecg showing sinus rhythm

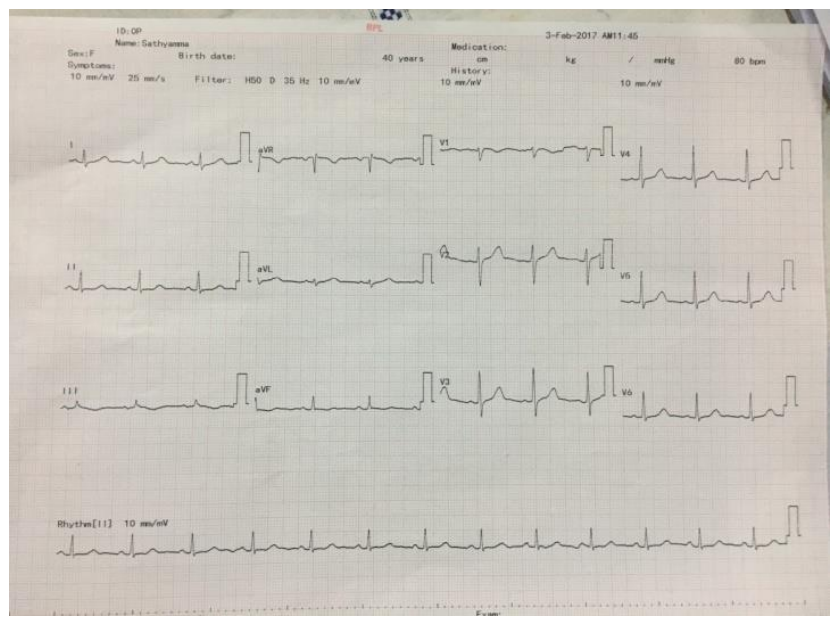

After 10 days of poisonous tree extract ingestion. In view of a possible digitalis intoxication, serum digoxin levels were measured which were $6 \mathrm{ng} / \mathrm{ml}$ (normal range: 0.8 to $2 \mathrm{ng} / \mathrm{ml}$ ).

On detailed examination of the leaves the ones that the patient had consumed, which the patient attenders brought, it was identified to be the leaves of Cryptostegia Grandiflora also called as the Indian rubber wine which is the source of cardiac glycosides (Fig. 5)

Fig 5. Leaves of Cryptostegia Grandiflora (Indian rubber vine, Kauli)

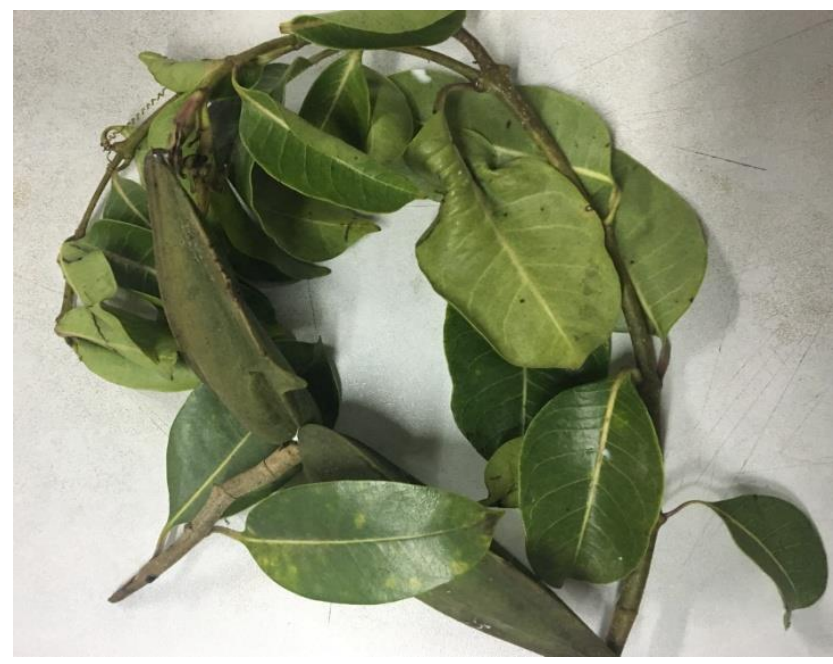

The patient was discharged on the $10^{\text {th }}$ day of admission with no complaints. 


\section{DISCUSSION :}

This is the second case report of Cryptostegia grandiflora in recent times. Cryptostegia grandiflora (Indian rubber vine) is a perennial climber widely distributed in India, Madagascar, Australia and Florida.

Its medicinal uses are as purgative, analgesic, wound healing remedies, antioxidant, antiviral, and for the treatment of schistosomiasis [2]. It is known in India by several local names like Vilayti-vakundi (Marathi), Garudappalai, Kauli (Marathi), Rubber vine, Chabukchhuree, Purple allamanda, Palai (Tamil), Palay Rubbervine, Rubber ki bel (Hindi). Our patient had consumed the plant for suicidal purpose. Leaves of Cryptostegia grandiflora, contain many cardiac glycosides (oleandrigenin, cryptostigmin I-IV) along with two cardelonides.1 Powdered leaves, mixed with water, when swallowed can cause persistent vomiting after half an hour; death in 15 hours. It is a poisonous plant causing death in cattle, sheep, goats and horses and hence is unpalatable and rarely eaten by them [3]. Other plant sources of cardiac glycosides include foxglove, yellow oleander, lily of the valley, wallflower and frangipani [1].

Cardiac glycosides reversibly inhibit the sodiumpotassium adenosine triphosphatase exchanger (Na-KATPase) in cardiac myocytes resulting in higher than normal intracellular concentrations of sodium and subsequently calcium, leading to positive inotropy. In toxicity, the influx of sodium increases phase IV depolarization, lowers the resting membrane potential threshold, and increases automaticity leading to its proarrhythmic potential. Although AV block and extrasystoles are the most common dysrhythmias seen, almost any dysrhythmia is possible. 1 in this case patient initially developed supraventricular tachycardia followed by complete heart block. Hyperkalemia is a major manifestation of cardiac glycoside toxicity and is a result of the inhibition of $\mathrm{Na}$ K-ATPase and the subsequent increase in extracellular potassium. In acute toxicity, there is a strong correlation between the degree of hyperkalemia and mortality from digoxin overdose[1]. Hyperkalemia was not seen in our patient. Na-K-ATPase exchangers are not limited to cardiac myocytes and systemic toxicity is commonly observed in addition to cardiac toxicity. Neurologic symptoms (independent of those caused by circulatory collapse) include confusion, weakness, lethargy, delirium, and disorientation. Gastrointestinal problems, such as nausea, vomiting, and anorexia, are also common [1]. Our patient presented with circulatory collapse with nausea, vomiting, lethargy and disorientation. Intravenous calcium has traditionally been considered contraindicated in digoxin overdose because hypercalcemia potentiates digoxin toxicity since theoretically, a non-contractile state is produced due to a failure of diastolic relaxation. Digoxin-specific antibody fragments (DSFab) is the treatment of choice and rapidly reverses cardiac manifestations of toxicity. Beta blockers may be considered for supraventricular tachyarrhythmias with a rapid ventricular response but may potentiate AV blockade, so short-acting agents, such as esmolol, are best. Atropine may be considered a temporary adjunct to treatment with DSFab, as is cardiac pacing [1]. Our patient did not receive digoxin-specific antibodies due to financial reasons, however, he responded well to symptomatic treatment and temporary pacemaker. There is evidence of thrombin and plasmin-like activities present in the latices of Cryptostegia grandiflora leading to its fibrinogenolytic and procoagulant action [4]. These properties can be responsible for the bleeding manifestation (epistaxis) but they were not seen in our patient. However, there are no reports of Cryptostegia grandiflora being responsible for the thrombocytopenia which was seen in our patient. The likely cause could be an immune thrombocytopenia secondary to its ingestion. A similar case of digitalis toxicity being produced by Cryptostegia grandiflora consumption has been documented by Mathur et al. and S A Single et al. [5 \& 7]. Severe cardiac arrhythmias have been described in the poisoning of ruminants by plants containing cardiac glycosides. Necropsy findings include evidence of congestive heart failure, epicardial and endocardial hemorrhages and focal myocardial necrosis with mononuclear inflammatory cell infiltrates and evidence of early fibroplasias [6]. Evidence of thrombocytopenia has been documented previously in one study, as was seen in our patient. Thus, in a patient of unknown poisoning presenting with circulatory collapse, GI disturbances, dysrhythmias and thrombocytopenia, one should suspect cardiac glycoside containing plant 
poisoning which needs to be treated promptly to reduce the high mortality rates associated with it.

\section{REFERENCES:}

1. Salmaan Kanji, Robert D. MacLean. Cardiac Glycoside Toxicity: More Than 200 Years and Counting. Crit Care Clin 2012; 28:527- 535.

2. CO Adewunmi. Natural Products as Agents of Schistosomiasis Control in Nigeria: A Review of Progress. Pharm Biol 1984; 22:161-16.

3. Austin DF. 1998. Poisonous Plants of Southern Florida

4. Viana CA, Oliveira JS, Freitas CD, et al. Thrombin and plasmin-like activities in the latices of Cryptostegia grandiflora and Plumeria rubra. Blood Coagul Fibrinolysis 2013;24:386-92.
5. Mathur KS, Dube BK, Kumar P. Cryptostegia grandiflora poisoning simulating digitalis toxicity. J Indian Med Assoc 1964; 42:381-5.

6. Cook DR, Campbell GW, Meldrum AR. Suspected Cryptostegia grandiflora (rubber vine) poisoning in horses. Aust Vet J 1990; 67:344.

7. Shashikala A Sangle, Sonali Inamdar, Vikrant Deshmukh. Cryptostegia grandiflora Toxicity Manifesting as Hyperkalemia, Complete Heart Block and Thrombocytopenia. Journal of The Association of Physicians of India2015;63. 